\title{
DESIGN, TESTING, AND COMMISSIONING OF AN ELECTROMAGNETIC UNDULATOR FOR SRC ${ }^{*}$
}

\author{
M.A. Green", W.R. Winter, M. Thikim, C.A. Baumann ${ }^{+}$, M.V. Fisher, \\ G.C. Rogers, D.E. Eisert, W.S. Trzeciak, R.A. Bosch \\ Synchrotron Radiation Center, 3731 Schneider Dr., Stoughton, WI 53589-3097
}

\section{Abstract}

In collaboration with one of its user groups, the SRC has installed a new planar electromagnetic undulator (EMU) on the Aladdin storage ring. Field quality, and resulting radiation-performance of the device, are sufficiently close to the ideal that no shimming was required. With slight exception, only minor, anticipated adaptation onto the Aladdin ring was needed to meet strict operational requirements. Major details of the design, fabrication, ex-citu and in situ testing, and commissioning are presented.

\section{DESIGN}

The EMU described here serves as a source for a high-resolution normal-incidence monochromator (NIM) beamline that covers the range from 6-40 eV [1]. The device was designed by SRC and constructed jointly with the Physical Sciences Laboratory (UW-Madison). The electromagnetic type was chosen for a number of reasons: favorable experience with a similar device for Iowa State University [2]; excellent field-quality control made possible with a copperiron geometry; and conventional construction that both uses inexpensive materials and enables in-house fabrication, leading to substantial cost savings.

\subsection{Mechanical Design}

The EMU operates in an environment for which the air temperature is $72{ }^{\circ} \mathrm{F}$ and the cooling water temperature is $95^{\circ} \mathrm{F}$. The elevated cooling temperature is used for all ring magnets and is related to the capacity of external cooling towers.

The pole material is 1006 hot-rolled, low-carbon steel. Each poleface surface is passivated against corrosion with an acrylic spray coating. The poles are affixed to 3"-thick intermediate, aluminum backing plates which in turn are bolted to upper and lower aluminum spars. Each spar assembly is supported at points which minimize spar deflection under combined gravitational and magnetic loading. Poles were dimensionally sorted, using an optical comparator, to achieve the greatest period uniformity. Stick micrometer measurements were made on the pole assemblies to verify positional accuracy. There is an observed increase in field, corresponding to a decrease in gap, over the center section of the device by $0.4 \%$ at zero field and $0.5 \%$ at full

* Work Supported by NSF Grant DMR-95-31009

\# E-mail: mike@src.wisc.edu

+ Physical Sciences Laboratory, 3725 Schneider Dr., Stoughton, WI excitation.

The coordinate system is right-handed with: $\mathrm{x}=$ transverse radially outward in the horizontal plane, $y=$ vertically "up", and z= longitudinal in the direction of the beam. The EMU is mounted on the ring from the outside such that the $+\mathrm{x}$-direction is toward the C-frame. Because of Aladdin's low injection energy of $108 \mathrm{MeV}$, the entire EMU is radially retractable on a motor-driven under-carriage as an additional safeguard against interference with injection.

Nominal mechanical and magnetic parameters are shown in Table 1.

Table 1: Mechanical \& Magnetic Parameters

\begin{tabular}{|l|c|}
\hline \multicolumn{1}{|c|}{ Parameter } & Value \\
\hline \hline Magnet Gap, g & $24 \mathrm{~mm}$ \\
\hline Pitch, $\lambda_{\mathrm{u}}$ & $10.9 \mathrm{~cm}$ \\
\hline Pole Width & $10.0 \mathrm{~cm}$ \\
\hline End Termination Pattern (w/o signs) & $\begin{array}{c}1 / 4,3 / 4,1,1 \ldots \\
\ldots 1,3 / 4,1 / 4\end{array}$ \\
\hline Total Number of Poles & 64 \\
\hline Overall Length & $3.5 \mathrm{~m}$ \\
\hline Peak Field & $4.0 \mathrm{kG}$ \\
\hline Peak K-value & 4.1 \\
\hline
\end{tabular}

\subsection{Electrical Design}

Nominal electrical parameters are shown in Table 2.

Table 2: Electrical Parameters

\begin{tabular}{|l|c|}
\hline \multicolumn{1}{|c|}{ Parameter } & Value \\
\hline \hline Total Inductance & 0.011 Henry \\
\hline Current at Peak Field & $257 \mathrm{~A}$ \\
\hline Voltage at Peak Field & $108 \mathrm{~V}$ \\
\hline Total Ohmic Power at Peak Field & $27.8 \mathrm{~kW}$ \\
\hline Power Supply Regulation & $\leq 0.4 \mathrm{~mA}$ \\
\hline Peak Current Density in Copper & $5070 \mathrm{~A} / \mathrm{in}^{2}$ \\
\hline
\end{tabular}

The power supply used for operation is DCCT-regulated and capable of 300-A by $120-\mathrm{V}$ output. Reflecting the onset of saturation, the required peak current at peak field is 
$3.5 \%$ more than expected from lower-excitation linear extrapolation.

The upper and lower pole assemblies are not magnetically "strapped" together [3]. In this geometry any environmental $\mathrm{B}_{\mathrm{y}}$ field is enhanced by roughly a factor of 2.5 resulting in an average $B_{y}$ field of about $1 \mathrm{G}$ from Earth's field. This field component is corrected by full-length coils around the upper and lower pole assemblies. Trim coils were provided on the first and last two poles (top and bottom), although their use has not been found necessary. Although also found unnecessary, passive current shunting of individual pole pairs (top \& bottom) was considered for phase shimming.

\section{FIELD MEASUREMENTS}

\subsection{Description}

Environmental The EMU was tested in an environmentally controlled room, for which the temperature could be stabilized at the ring operating temperature of $72{ }^{\circ} \mathrm{F}$. Similarly, a closed-loop, thermally-regulated source of cooling water at $95{ }^{\circ} \mathrm{F}$ was also provided. Under these conditions stabilized temperatures over the upper and lower aluminum spars varied by $\pm 0.2^{\circ} \mathrm{F}$ and $\pm 0.7^{\circ} \mathrm{F}$, respectively.

Control \& Data Acquisition The mapping system is composed of a Pentium-based PC with a stepping motor controller board, a GPIB board and a 16-bit ADC/DAC board. Two stepping motors are used by the system. One motor drives a Group 3 Hall probe longitudinally (z) through the undulator gap and the second motor positions the probe transversely (x). An on-axis laser interferometer is used to measure the probe's relative longitudinal position in the undulator. A digital micrometer is used at one end of the undulator to set/reset the initial absolute position. During mapping the laser interferometer issues TTL pulses every $25 \mu \mathrm{m}$ which trigger the ADC board to read the analog output from the Hall probe.

The mapping system collects 180000 data points on both forward (-z) and return $(+\mathrm{z})$ passes. At the end of the forward pass the Hall probe enters, and briefly stops within, a magnetic shield where the software records 24000 background data points at about $8000 / \mathrm{sec}$. A complete round trip of a forward pass, a background measurement, and the return pass takes about one minute. At this probe speed the peak data rate is also about $8000 / \mathrm{sec}$. The software can make a series of round trip passes at preprogramed radial (x) displacements without user intervention.

Apparatus The analog output from the G3 Hall probe was precalibrate at the factory. The probe is supported on a carriage that has a manual y-motion stage and the computercontrolled $\mathrm{x}$-motion stage. The carriage moves at a rate of about $200 \mathrm{~mm} / \mathrm{sec}$ along a $4.5-\mathrm{m}$ rail that extends about $0.5 \mathrm{~m}$ past each end of the EMU. Limit switches for the longitudinal carriage drive are provided in the event of computer-drive error. The rail is supported off the lower spar keeping the probe at the same relative vertical position with respect to the median plane for a fixed-gap device. A retroreflector travels with the carriage along the $\mathrm{z}$-axis such that laser interferometer measurements are not affected by offset errors. Refractive index air-path corrections were made for the interferometer measurements. Because of the elevated operating temperature, it was necessary to create turbulence in the air along the longitudinal axis for proper operation of the interferometer.

The probe is mounted in a holder which allows arbitrary $90^{\circ}$ rotations about the $\mathrm{z}$-axis allowing $\mathrm{B}_{\mathrm{y}}, \mathrm{B}_{\mathrm{x}}$, and flipped $-B_{y},-B_{x}$ measurements. Measurements taken in the fieldshielded region (between the forward and reverse passes) allowed determination of the Hall probe offset, the residual background, and any magnetization of the shield, which values were subsequently factored into the data analysis. No significant dependence of these parameters was found either on x-position or on device excitation.

It must be noted that the maximum excitation for testing was $240 \mathrm{~A}$ whereas that for operation is $257 \mathrm{~A}$. The difference arises because the new power supply for operation had not been delivered in time for testing.

\subsection{Results}

Hysteresis As standard procedure, the device was always operated on a standard uni-polar hysteresis curve, attained by cycling from minimum to maximum to minimum excitation 5 times. The maximum difference between the lower and upper branches was about $15 \mathrm{G}$ in field, or about $0.8 \%$ in relative photon energy.

Multipoles Simple summation of the 180000-point $\mathrm{B}_{\mathrm{y}}(\mathrm{x})$ or $\mathrm{B}_{\mathrm{x}}(\mathrm{x})$ data files result in the longitudinal integrals $\mathrm{IB}_{\mathrm{y}}(\mathrm{x}) \equiv \int \mathrm{B}_{\mathrm{y}}(\mathrm{x}, \mathrm{z}) \mathrm{dz}$ and $\mathrm{IB}_{\mathrm{x}}(\mathrm{x}) \equiv \int \mathrm{B}_{\mathrm{x}}(\mathrm{x}, \mathrm{z}) \mathrm{dz}$, respectively, all additionally as functions of the excitation. It is felt that rms errors in the IB's do not exceed about $1 \mathrm{G} \cdot \mathrm{cm}$. From the IB's, the respective normal and skew multipole moments are derived by fitting. Over all excitations, the required and

Table 3: Multipole Magnitudes, Over All Excitations

\begin{tabular}{|ll|c|c|c|}
\hline \multicolumn{2}{|c|}{ Multipole } & Req'd & Meas. & Units \\
\hline \hline \multirow{2}{*}{ Dipole } & Normal & $100 \cdot 10^{-6}$ & See Text & \\
& Skew & $100 \cdot 10^{-6}$ & $170 \cdot 10^{-6}$ & $\mathrm{~T} \cdot \mathrm{m}$ \\
\hline \multirow{2}{*}{ Quadrupole } & Normal & $6.7 \cdot 10^{-3}$ & $0.9 \cdot 10^{-3}$ & \\
& Skew & $1 \cdot 10^{-3}$ & $0.6 \cdot 10^{-3}$ & $\mathrm{~T}$ \\
\hline \multirow{2}{*}{ Sextupole } & Normal & 1.25 & 0.03 & $\mathrm{~T} / \mathrm{m}$ \\
& Skew & 1.25 & 0.03 & \\
\hline \multirow{2}{*}{ Octupole } & Normal & 40 & 5 & $\mathrm{~T} / \mathrm{m}^{2}$ \\
& Skew & 40 & 4 & \\
\hline
\end{tabular}


measured multipoles are as shown in Table 3. Since a longcoil is present to correct $\mathrm{IB}_{\mathrm{y}}$, the normal-dipole component is variable; a single, fixed setting for this coil was found which satisfied the requirement. The skew-dipole (vertical steering error) is out of the original spec but is not so easily corrected. However, this component has been relaxed since the ring orbit control is quite able to cope with this steering error.

\% Ideal The on-axis $\mathrm{B}_{\mathrm{y}}(0,0, \mathrm{z})$ and $\mathrm{B}_{\mathrm{x}}(0,0, \mathrm{z})$ fields are used to predict and assess the quality of the undulator radiation that would be emitted, using the UR code [4]. There are two systematic field errors which are known to affect the harmonic radiation in the present case. The first can be from the concentration of Earth's field, if the long $\mathrm{B}_{\mathrm{y}}$ coil isn't used, and the second stems from the mid-section field enhancement. With fixed, long-coil correction active, degradation of the $3^{\text {rd }}$ harmonic over its useful range is computed to be less than $10 \%$, the majority of which is not due to the mid-section systematic error.

Internal Orbit Walk Vertical and horizontal orbit integrations interior to the undulator affect both the quality of the radiation (see above) and the effective source point of radiation for the beamline. Over all excitations, the variation of effective source point is $\leq 30 \mu \mathrm{m}$ after gross steering correction.

Phase Errors A common measure of the field quality that affects the quality of radiation is rms phase error [5]. At selected values over the range of excitations, this value ranges from $1.0^{\circ}$ at low excitation to $4.6^{\circ}$ at high excitation.

\section{CONTROL}

Quad Shunting The undulator is situated in a long straight section between two FODOF triplets. A natural consequence of undulator operation is vertical edge focusing. SRC policy is presently to limit any dynamic, undulator-induced relative vertical beam size variations around the ring to $\leq 1 \%$. If the additional vertical focusing were left uncorrected, the resulting relative vertical beam size changes would be excessive, thus necessitating correction. An approximate, yet satisfactory, means of correction is to shunt current in adjacent, upstream and downstream, quadrupoles. The degree of shunting required is nearly proportional to $\mathrm{K}^{2}$ and is easily programmed into the control system.

BPM Offsets Aladdin has horizontal and vertical feedback control of the closed orbit. In the straight section containing the undulator there are basically four beam-position-monitor (BPM) and steering-magnet combinations in both vertical and horizontal planes with inner and outer pairs straddling the undulator. The inner-pair BPM-steerer combinations can have preset orbit offsets for position and angle steering of the undulator radiation source for alignment into the attendant beamline. This done, the outer pairs maintain an unperturbed closed orbit everywhere else around the ring.

Hysteresis Operationally, a possible $0.8 \%$ energy uncertainty from hysteresis was considered unacceptable. Therefore, a Hall probe was permanently installed on a pole-face and calibrated against the on-axis field. Precision of calibration is not very important (its within a few tenths of an $\mathrm{eV}$ ) but the repeatability that this solution makes possible is. Software feedback control of the undulator's excitation is entered by the beamline user in $\mathrm{eV}$ and relies upon the Hall probe for its operation.

\section{INITIAL EXPERIENCE}

The EMU is now installed on the Aladdin ring where it is also paired-up with the new power supply. SRC maintains the option of installing magnetic shielding between any insertion device and neighboring quadrupoles. So far this hasn't been necessary, the present case in particular. Preliminary ambient-field mapping in the straight section where the device is installed was conducted, resulting in the correction of a very minor problem with an ion pump.

The radial retraction of the undulator for low-energy injection also is found empirically unnecessary. In fact, actually discovered by accident, injection is observed to proceed with little difficulty (with only steering correction) at $70 \mathrm{~A}$ of excitation.

Without quad-shunt correction, the observed relative vertical beam size changes at optical monitors around the ring are $\leq 8 \%$. However, with quad-shunt correction the observed vertical beam size variations at the optical monitors are $\leq 1 \%$ over the full operating range of excitations. Operationally, any beam positional variations around the ring, in the presence of global feedback orbit control, are entirely satisfactory. Further, no adverse coupling or lifetime effects have been observed.

Lastly, the attendant high-resolution NIM beamline is just now being commissioned. Consequently, comparative measurements and experience from the beamline are not yet available. It is anticipated that these will be reported in the near future.

\section{REFERENCES}

[1] R. Reininger, et al., Rev. Sci. Instrum. 66, 2194 (1995).

[2] F.C. Younger, W.J. Pearce, B. Ng, Nucl. Instr. and Meth. in Phys. Res. A 347, 98 (1994).

[3] Decided after private correspondence with Ross Schlueter (LBL).

[4] R.J. Dejus and A. Luccio, Nucl. Instr. and Meth. in Phys. Res. A 347 (1994) 61.

[5] R.J. Dejus, I. Vasserman, E.R. Moog, E. Gluskin, Rev. Sci. Instrum. 66, 1875 (1995). 